\title{
Neither repetition duration, nor number of muscle actions affect strength increases, body composition, muscle size or fasted blood glucose in trained males and females
}

\begin{tabular}{|c|c|}
\hline Journal: & Applied Physiology, Nutrition, and Metabolism \\
\hline Manuscript ID & apnm-2018-0376.R1 \\
\hline Manuscript Type: & Article \\
\hline $\begin{array}{r}\text { Date Submitted by the } \\
\text { Author: }\end{array}$ & 05-Jul-2018 \\
\hline Complete List of Authors: & $\begin{array}{l}\text { Carlson, Luke; Discover Strength, } \\
\text { Jonker, Brandon; Discover Strength } \\
\text { Westcott, Wayne; Quincy college, Exercise Science } \\
\text { Steele, James; Southampton Solent University, Centre for } \\
\text { Health, Exercise and Sport Science } \\
\text { Fisher, James; Southampton Solent University, Centre for } \\
\text { Health, Exercise and Sport Science }\end{array}$ \\
\hline $\begin{array}{r}\text { Is the invited manuscript } \\
\text { for consideration in a } \\
\text { Special Issue? : }\end{array}$ & Not applicable (regular submission) \\
\hline Keyword: & lean mass, superslow, velocity, body fat \\
\hline
\end{tabular}


Neither repetition duration, nor number of muscle actions affect strength increases, body composition, muscle size or fasted blood glucose in trained males and females

Luke Carlson ${ }^{1}$, Brandon Jonker ${ }^{1}$, Wayne L. Westcott ${ }^{2}$, James Steele ${ }^{3,4}$, James P. Fisher ${ }^{3}$.

${ }^{1}$ Discover Strength, $101606^{\text {th }}$ Avenue North, Suite A, Plymouth, MN 55441, USA

2 Quincy College, Quincy, Massachusetts, USA

${ }^{3}$ School of Sport, Health and Social Science, Southampton Solent University, UK

${ }^{4}$ ukactive Research Institute, ukactive, London, UK

Corresponding Author:

James Peter Fisher

School of Sport, Health and Social Science

Southampton Solent University

East Park Terrace

Southampton

UK

Tel: +44 2382013163

Email: james.fisher@solent.ac.uk

Co-Author contact details:

Luke Carlson (Luke@discoverstrength.com)

Brandon Jonker (jonkerb@gmail.com)

Wayne L. Westcott (wwestcott@quincycollege.edu)

James Steele (James.steele@solent.ac.uk)

Word Count: 4997

Abstract: 236 
Repetition Duration in trained males and females

\section{Abstract}

A key variable within resistance training (RT) is that of repetition duration; the time (seconds; s) taken to perform the concentric and eccentric muscle actions of a repetition. Research has produced equivocal results with regards to strength and muscle mass increases whilst many studies have created parity in the number of repetitions though there has been a disparity in load used and timeunder-load (TUL). The purpose of this study was to compare load, and TUL matched groups performing resistance exercise using differing repetition durations. Fifty-nine male and female participants were randomised in to 3 groups; $2 \mathrm{~s}: 4 \mathrm{~s}(n=18), 10 \mathrm{~s}: 10 \mathrm{~s}(n=20)$ and a group which performed a 30s eccentric, 30s concentric and 30s eccentric muscle actions (e.g. 1.5 repetitions; $n=21$ ). Participants were supervised in 1-on-1 RT sessions $2 \mathrm{~d} \cdot \mathrm{wk}^{-1}$ for 10 weeks. Outcomes were 10repetition maximum (RM) and predicted 1RM for chest press, leg press and pull-down exercises, as well as body composition, upper arm and thigh muscle mass and fasted blood glucose. Analyses revealed significant increases in strength for all exercises but no between-group differences, and no statistically significant time course changes for the other variables. Repetition duration does not affect the increases in strength in trained participants where exercise is performed to momentary failure. Since time constraints and perceived difficulty are often cited barriers to exercise, it is important to recognise that the low-volume (single-set), machine-based protocol employed herein produced worthwhile strength increases in trained participants.

Key words: lean mass, superslow, velocity, body fat, 


\section{Introduction}

The health benefits of resistance training (RT) are now well recognised with recent calls being made to make RT a more prominent part of public health messaging around physical activity and exercise (Steele et al. 2017b). Numerous variables are often manipulated within a RT programme such as load (Fisher et al. 2017, 2017b), volume (Barbalho et al. 2017; Baker et al. 2013; Schoenfeld et al. 2017), frequency (Schoenfeld et al. 2015), intensity of effort (Steele et al. 2017; Fisher et al. 2016), muscle actions (Fisher et al. 2016b), and use of advanced training techniques (Fisher et al. 2015; Fisher et al. 2014).

However, an area which has received limited consideration is that of repetition duration. This is generally reported as the time duration (seconds) for concentric and eccentric muscle actions. For example, a 2 second concentric phase, and 4 second eccentric phase may be represented as; $2 s: 4 s$. The force-velocity curve supports that higher forces are attained at slower speeds as a result of an increasing number of simultaneously contracting muscle fibres due to a greater number of cross-bridge attachments permitted by the slower muscle shortening (Wilkie 1950). For example, a longer repetition duration would allow greater time for crossover of the actin and myosin filaments within a muscle, meaning a higher number of cross-bridges are formed and maintained throughout the muscle action. As such, a slower movement/higher repetition duration would theoretically increase mechanical tension throughout an exercise which is hypothesised to positively facilitate intracellular anabolic signalling, which, in turn, might be a driving factor towards greater hypertrophic adaptation (Schoenfeld 2010). Furthermore, continuous force generation at $>40 \%$ maximum voluntary contraction has been shown to increase intramuscular pressure, creating shortterm ischaemia to the muscle (Bonde-Peterson et al. 1975). By creating this temporary hypoxic condition there is thought to be greater increases in muscle size and strength by promoting recruitment of fast-twitch muscle fibres as a result of increasing fatigue and metabolic stress (e.g. blood lactate, hydrogen ions, and inorganic phosphate) which, in turn, might stimulate an elevation in human growth hormone (hGH) response (Schoenfeld 2010; Loenneke et al. 2010). 
Repetition Duration in trained males and females

In support, Tanimoto and Ishii (2006) and Tanimoto et al. (2008) reported greater muscle deoxygenation during resistance exercise using a longer-repetition duration and lighter load (LST; 3s concentric: 1s isometric pause: 3s eccentric @ 50-60\% 1 repetition maximum; RM) compared to shorter- repetition durations with heavier- and lighter- loads (HN; 1s:1s @80-90\% 1RM, and LN; 1s:1s @50\% 1RM, respectively). Furthermore, significantly greater blood lactate concentration values occurred in both the HN and LST groups compared to the LN group (Tanimoto and Ishii 2006), with both the HN and LST groups showing similar improvements in strength and hypertrophy (Tanimoto and Ishii 2006; Tanimoto et al. 2008). However, time-under-load (TUL) differed between groups with each performing 8 repetitions. Therefore, the LST group performed $\sim 56 \mathrm{~s}$ total TUL, whilst the HN and LN groups performed 16s TUL. In a similar study design using older adults comparing LST (3s:1s:3s) and LN (1s:1s) at 50\% 1RM, Watanabe et al. (2013) reported elevated blood lactate levels along with greater muscle hypertrophy of the quadriceps following longer- compared to shorter- repetition duration resistance exercise. Once again though TUL differed between groups ( $\sim 6 s$ compared to 16s). Finally, Goto et al. (2008) reported elevated norepinephrine and free testosterone levels following longer repetition duration resistance exercise (3s:1s:3s at $40 \% 1 \mathrm{RM})$ compared to traditional repetition duration exercise (1s:1s at $80 \%$ and $40 \% 1 \mathrm{RM})$. In combination, these suggest that longer repetition duration resistance exercise might produce lower peripheral muscle oxygenation levels during exercise, and increase blood lactate concentration and circulating anabolic hormones post-exercise. However, strength and hypertrophic adaptations for groups using a longer repetition duration (3s:1s:3s) at lighter-loads (e.g. 40-60\% 1RM) were similar to groups using a shorter repetition (1s:1s) and a heavier load (e.g. 80-90\% 1RM). In this sense, it is worth noting that a growing body of research is supporting that acute post-exercise hormonal rises are not related to chronic changes in muscle mass or strength (West, et al. 2012; Morton, et al. 2016). As such the favourable adaptations for longer repetition duration or heavier load might be related to other factors such as muscle fibre, and corresponding motor unit, recruitment (Denny-brown and Pennybacker 1938; Carpinelli, 2008; Fisher, et al. 2017). 
A further acute study reported higher myofibrillar protein synthetic fractional rate, which correlated to p70S6K phosphorylation, in a slow- (6s:6s) compared to a control- (1s:1s) group for unilateral knee extension exercise (Burd, et al. 2012). However, the research design used a load equating to $30 \% 1 \mathrm{RM}$, and only the slow group performed repetitions to MF, whilst the control group performed an equivalent number of repetitions not to MF. In this sense, the study repeats the previous limitations of not equating TUL and exercising to MF, and as such whilst the longer TUL provided greater stimulation for markers associated with muscle growth, this is not reflective of a longer repetition duration.

Further evidence of positive adaptations resulting from even longer repetition durations are presented by Fisher et al. (2014b), who reported very large effect sizes (ESs) and relative strength increases (38.4\% to $90.8 \%$ ) for older adults, using a 10 s:10s repetition duration for pull-down, chest press, seated row, overhead press and leg press exercises. Furthermore, Westcott et al. (2001) compared SuperSlow training groups (10s:4s) to traditional training groups (2s:1s:4s) training for 8or 10 -weeks across two studies showing approximately $50 \%$ greater strength increases $(p<0.001)$ for the $10 \mathrm{~s}: 4 \mathrm{~s}$ repetition duration in previously untrained participants.

More recent research has reported equivocal findings. Keeler et al. (2001), Rana et al. (2008) and Schuenke et al. (2012) ${ }^{1}$, and Kim et al. (2011) all compared SuperSlow - (SS) to traditional- (TR) repetition duration RT. However, a notable limitation is the disparity in loading patterns TUL between groups. Where parity was maintained in the number of repetitions, and thus concentric and eccentric muscle actions, the groups using a longer repetition duration used a lighter load and thus exercised for a longer TUL (Keeler et al. 2001; Rana et al. 2008; Kim et al. 2011). The use of a heavier-load for some groups might have caused a more favourable strength adaptation as a result of specifically practicing lifting a heavier load (Fisher et al. 2017; Mattocks et al. 2017). In addition,

\footnotetext{
${ }^{1}$ Rana et al. (2008) and Schuenke et al. (2012) were actually the same study but the authors reported strength and morphological adaptations in the different, respective publications.
} 
Repetition Duration in trained males and females

research has suggested that the use of a lighter load/longer TUL might cause a greater degree of discomfort (Fisher et al. 2017b) which, for the SuperSlow groups, might have resulted in participants ceasing exercise before reaching momentary failure (Fisher et al. 2017).

To date, other than completely isometric training, where there is no change in the joint angle and thus no concentric or eccentric muscle actions, no studies appear to have considered repetition durations longer than 10s:10s, and any results only apply to comparisons where repetition number, rather than load and/or TUL, have been matched between groups. Therefore, the present study was designed to consider the effects of three different, load and TUL matched, repetition duration protocols; controlled (2s:4s) SuperSlow (10s:10s) and a single 30s concentric muscle action preceded and succeeded by 30 s eccentric muscle actions (30s eccentric: 30 s concentric: $30 \mathrm{~s}$ eccentric) upon strength, body composition, upper arm and thigh muscle cross-sectional area (CSA). Furthermore, whilst elevated fasted blood glucose (e.g. $>85 \mathrm{mg} / \mathrm{dl}$ ) can be a risk factor for cardiovascular death (Bjørnholt et al. 1999) no studies appear to have considered the impact of resistance training on fasted blood glucose in healthy, trained participants. As such, within the present study fasted blood glucose levels were also measured pre- and post-intervention.

\section{$\underline{\text { Methods }}$}

\section{$\underline{\text { Study Design }}$}

A randomised controlled trial design was adopted, with three experimental groups included. The effects of three RT interventions utilising varying repetition duration manipulations while controlling load were examined in trained participants upon muscular performance and body composition. The study design was approved by the relevant ethics committee at the corresponding author's institution (REF: FISHER190218).

\section{$\underline{\text { Participants }}$}


Sixty-two participants (males $=26$, females $=36$ ) were recruited from the present membership pool in a USA strength training facility (Discover Strength, Minnesota). All participants were required to have had $>6$ months RT experience at the present facility incorporating low volume (single set) RT to momentary failure (MF) for most major muscle groups $\sim 2 \mathrm{~d} \cdot \mathrm{wk}^{-1}$. All participants were currently physically active (e.g. walking, jogging, cycling, swimming, or yoga) outside of participation in RT and were instructed not to change their physical activity habits or to begin any other structured exercise programs (with the exception of the RT interventions examined in the present study). Participants were also free from any medical condition for which RT is contraindicated. Written informed consent was obtained from all participants prior to any testing and group allocation was performed by computer randomisation to one of three groups; controlled repetition duration ('2s:4s'), slow repetition duration ('10s:10s'), or a very slow repetition duration ('30s:30s:30s').

Three dropouts occurred during the study. One male dropped out of the 30s:30s:30s group due to dislike of the protocol, and two females dropped out of the $2 \mathrm{~s}: 4 \mathrm{~s}$ groups, one which was lost to follow up and the other due to becoming pregnant during the study. As such, final group numbers were: $2 \mathrm{~s}: 4 \mathrm{~s}, n=18($ males $=7$, females $=11), 10 \mathrm{~s}: 10 \mathrm{~s}, n=20($ males $=10$, females $=10)$, and 30s:30s:30s, $n=21$ (males $=8$, females $=13$ ).

\section{Procedures}

\section{Testing}

Pre- and post-intervention muscular performance testing was performed using an estimated 10 repetition maximum (RM) from which $1 \mathrm{RM}$ was predicted. This was performed in the following order with 120 seconds rest between exercises using chest press (Nautilus Evo, Nautilus, Vancouver, Washington, US), leg-press (Avenger, MedX, Ocala, Florida, USA) and pull-down² (MedX, Ocala, Florida, USA) resistance machines. Since all participants were existing members of the facility where

\footnotetext{
${ }^{2}$ Notably, the leverage of the pull-down machine combined with the seat position for a full range-of-motion on the leg press means that a heavier load is often lifted for the pull-down exercise.
} 
Repetition Duration in trained males and females

testing and training took place, 10RM loads were estimated from pre-existing training load for testing. Participants first performed a warm-up set of 5 controlled repetitions using a load equal to $50 \%$ of the estimated 10RM load. After 90 seconds rest the participants then performed a set of repetitions to MF using a 2 second concentric, 4 second eccentric repetition duration. If participants exceeded 10 repetitions, they were instructed to stop and not continue to MF. A rest of 5 minutes then permitted before adjusting the load and attempting the 10RM again. It was not considered essential that an exact 10RM load was identified, just that a load permitting failure between 7-10 repetitions was (for both pre and post testing this resulted in a mean of $\sim 7$ repetitions). This was because this test was then used to predict 1RM using the Brzycki (1993) equation (predicted 1RM = load lifted / (1.0278 - (0.0278 x number of repetitions $))$ which has been shown to have a very high correlation to actual 1RM ( $r=0.99$; Nascimento et al. 2007). It was considered that this method provides strong ecological validity to realistic training conditions as most people infrequently test or use their maximal strength. MF during testing was defined as the point where, despite the greatest effort, the participant failed to complete the concentric phase of a repetition (Steele et al. 2017). Post testing was performed at least 48 hours following the final training session as per previous research (Fisher et al. 2015; Fisher et al. 2014). The instructor performing the pre- and post-testing was blinded to group assignment. Predicted 1RM was considered for each exercise in addition to a combined strength total calculated as the sum of predicted 1RM from each exercise.

Body composition was estimated using both air displacement plethysmography (Bod Pod GS, Cosmed, Chicago, IL, USA) as well as anthropometric measures. Details of the test procedures for estimation of body composition using air displacement plethysmography have been previously described in detail elsewhere (Dempster et al. 1995). Briefly, whilst wearing minimal clothing (swimsuit or tight fitting underwear) and a swim cap, participants were weighed using a calibrated digital scale. The participant was then seated in the Bod Pod for body volume measurement. From the body mass and body volume measurements, and predicted thoracic lung volumes, body density was estimated by the Bod Pod software and lean and fat mass estimations calculated using the Siri 
equation. Estimates of both arm muscle area (MA) and thigh MA were made from anthropometric measurements using methods and equations described previously by Heymsfield et al. (1982) and Housh et al. (1995) respectively. Briefly, for arm MA this involved measurement of circumference at the midpoint between the tip of the acromion and olecranon process with the arm hanging relaxed by the participant's side and triceps skinfold taken as a vertical fold at the same point using callipers (Harpenden, Baty International, UK). For thigh MA a measurement of circumference was made at the midpoint of the inguinal crease and proximal border of the patella and a thigh skinfold taken as a vertical fold at the same point using the same callipers. All measurements were taken by the same instructor blinded to group assignment and at baseline anthropometric measures were performed twice with at least 48 hours between tests to determine typical error of measurement for arm MA and thigh MA using spreadsheets for reliability produce by Hopkins (2015) These were $2.76 \mathrm{~cm}^{2}$ $(95 \% \mathrm{Cl}=2.33$ to 3.38$)$ and $5.64 \mathrm{~cm}^{2}(95 \% \mathrm{Cl}=4.77$ to 6.91$)$ for arm MA and thigh MA respectively. Lastly, measurement of fasting blood glucose was made using an Accutrend Plus Glucometer (Roche, Switzerland). Capillary blood samples were taken in the morning after a minimum overnight fast of 8 hours. This was performed using the procedures outlined by Maughan et al. (2011). The tip of the participant's middle finger on their non-dominant hand was cleaned using an alcohol wipe and then allowed to air dry. The skin on the prepared area finger was then punctured using a disposable lancet and the first bolus of blood wiped away using a clean tissue. The next bolus of blood was sample using the glucometer strip.

Training Intervention (2s:4s, 10s:10s, 30s:30s:30s)

Training was performed $2 \mathrm{~d} \cdot \mathrm{wk}^{-1}$ (with at least 48 hours between sessions) for 10 weeks. Two workouts were performed (A \& B) for exercises included in table 1 for a single set and all groups used the same approximate relative load which was equal to the load determined from the pre estimated 10RM test. All training sessions were performed at a 1:1 (trainer: trainee) supervision ratio. All exercises were performed using Nautlius Evo or Nautilus 2ST (Nautilus, Vancouver, 
Repetition Duration in trained males and females

Washington, USA), MedX or MedX Avenger (MedX, Ocala, Florida, USA), or Hammer Strength (Rosemont, Illinois, USA) equipment

*Insert table 1 around here*

The 2s:4s group performed each exercise using a 2 second concentric and 4 second eccentric repetition duration. The 10s:10s group performed each exercise using a 10 second concentric and 10 second eccentric repetition duration. Both of these group continued repetitions to the point of MF as defined above (Steele et al. 2017). The 30s:30s:30s group began with the eccentric phase first after the instructor (with an additional instructor to assist if needed) lifted the movement arm into the end part of their range of motion. Participants then completed a 30 second eccentric, followed by a 30 second concentric, followed by a further 30 second eccentric, and then attempted a final concentric action to confirm that concentric MF had occurred. Timing was assessed by an ACSM qualified personal trainer using a stopwatch, and, in the case of the 30s:30s:30s; reported to the participant every $5 \mathrm{~s}$ in order to maintain a steady pace throughout the range of motion. The instructor also provided technical coaching to maintain proper form during the exercise. Every $4^{\text {th }}$ workout (e.g. sessions 4, 8, 12, 16, and 20) were performed using the same 2s:4s repetition duration used for 10RM testing to prevent specificity of repetition duration favouring the 2s:4s group. Furthermore, this session allowed assessment that parity in load was being maintained between all groups.

The loads being used permitted participants in each group to perform repetitions and time under load (TUL) as follows: the 2s:4s group performed between 8-12 repetitions (56-84 seconds TUL); the 10s:10s group performed between 3-5 repetitions (60-90 seconds TUL); and the 30s:30s:30s group performed 1.5 repetitions (90 seconds TUL). Once participants could perform more than the desired repetitions/TUL (e.g. $\geq 12$ repetitions/84 seconds for the $2 \mathrm{~s}: 4 \mathrm{~s}$ group; $\geq 5$ repetitions/90 seconds for the 10s:10s group; and 1.5/90 seconds for the 30 s:30s:30s group) load was increased by $\sim 5 \%$ as per previous recommendations and research (Fisher et al. 2015; Fisher et al. 2014). 


\section{Statistical Analyses}

Power analysis of research using low-volume RT in trained participants (Fisher et al. 2014) was conducted to determine participant numbers $(n)$ using an effect size (ES), calculated using Cohen's $d$ (Cohen 1992) of 1.25 for improvements in strength. Participant numbers were calculated using equations from Whitley and Ball (2002), revealing that each group required 9 participants to meet required $\beta$ power of 0.8 at an $\alpha$ value of $p \leq 0.05$. After dropouts $(n=3)$, data were available from 59 participants $(2 \mathrm{~s}: 4 \mathrm{~s}, n=18$ (males $=7$, females $=11), 10 \mathrm{~s}: 10 \mathrm{~s}, n=20$ (males $=10$, females $=$ 10), and 30s:30s:30s, $n=21$ (males $=8$, females $=13$ ).

The independent variable was 'group' and the dependent variables were the absolute change (post- minus pre-test) in each outcome variable. Analysis of Covariance (ANCOVA) was used to compare absolute change for each outcome variable between groups with pre-test values used as covariates for each analysis. Statistical analysis was conducted using JASP (version 0.8.1.2; University of Amsterdam, The Netherlands) with $\alpha$ for statistical significance set at 0.05 . Further, estimated marginal means with 95\% confidence intervals $(\mathrm{Cl})$ were calculated in order to determine whether significant within group changes had occurred. The researcher who performed the data analyses was blinded to group assignment.

\section{Results}

$\underline{\text { Participants }}$

Participant baseline demographics are shown in Table 2. Demographic variables did not differ between groups at baseline.

\section{*INSERT TABLE 2 ABOUT HERE*}

\section{Muscular Performance}

ANCOVA revealed no significant between-groups differences in change in predicted 1RM for either chest press $\left(F_{(1,55)}=2.633, p=0.081\right)$, leg press $\left(F_{(1,55)}=1.611, p=0.209\right)$, pulldown $\left(F_{(1,55)}=\right.$ 
$0.641, p=0.531)$, or total strength $\left(F_{(1,55)}=1.554, p=0.221\right)$. Table 2 shows estimated marginal means for change in predicted $1 \mathrm{RM}(\mathrm{kg})$ for each exercise and total strength. Significant withingroups changes in predicted 1RM occurred for each group in all exercises and for total strength as evidenced by the $95 \% \mathrm{Cls}$ not crossing zero (see Figure 1). Predicted $1 \mathrm{RM}$ values are provided for preand post-intervention for chest press, leg press and pull down exercises in table 2.

Body Composition

ANCOVA revealed no significant between-groups differences in change in body fat percentage $\left(F_{(1,54)}=0.394, p=0.676\right)$, lean mass, $\left(F_{(1,54)}=1.744, p=0.184\right)$, arm $\mathrm{MA}\left(F_{(1,53)}=1.212, p=\right.$ $0.306)$, or thigh $\mathrm{MA}\left(F_{(1,54)}=1.272, p=0.288\right)$. Table 3 shows estimated marginal means for change in body fat percentage, lean mass, arm MA and thigh MA. There were no significant within-groups changes for any of these outcome measures as evidence by the $95 \% \mathrm{Cls}$ crossing zero.

*Insert table 3 around here*

\section{Blood Glucose}

ANCOVA revealed no significant between-groups differences in change in fasting blood glucose $\left(F_{(1,52)}=0.381, p=0.685\right)$. Estimated marginal means and $95 \% \mathrm{Cls}$ for changes in fasting blood glucose were $-5.2 \mathrm{mg} / \mathrm{dL}(-12.2$ to 1.18$)$ for the $2 \mathrm{~s}: 4 \mathrm{~s}$ group, $-6.2 \mathrm{mg} / \mathrm{dL}$ (-13.6 to 1.1$)$ for the 10s:10s group, and $-1.7 \mathrm{mg} / \mathrm{dL}$ (-9.7 to 6.3$)$ for the $30 \mathrm{~s}: 30 \mathrm{~s}: 30 \mathrm{~s}$ group. There were no significant within-groups changes as evidenced by the $95 \%$ Cls crossing zero.

\section{Discussion}

The present study examined the effects of repetition duration upon strength, body composition, arm and thigh muscle mass and fasted blood glucose levels. To our knowledge this represents the only study to consider a very slow movement equating to only 1.5 repetitions; $30 \mathrm{~s}$ eccentric: 30 s concentric: 30 s eccentric in a load-matched comparison to $2 \mathrm{~s}: 4 \mathrm{~s}$ and $10 \mathrm{~s}: 10 \mathrm{~s}$ repetition durations. Our results suggest that all groups made significant increases in muscular 
strength across chest press, leg press and pull-down exercises with no between-group differences. In the first instance this is notable since many persons cite time-constraints and perceived difficulty as reasons for non-participation or poor adherence to exercise protocols (Trost, et al. 2002; Silliman, et al. 2004; Winett, et al. 2009). The low-volume (single-set), resistance machine based protocol performed herein produced meaningful strength increases even in trained participants in $\leq 1$ hour per week.

Our data suggests that strength increases are likely not affected by the repetition duration used but rather a product of exercising to a high degree of effort, e.g. reaching momentary failure. This is in accordance with previous research which has suggested a threshold with respect to RT intensity of effort (Fisher et al. 2016). Indeed, other studies have suggested that there are no greater strength increases acquired by training beyond momentary failure (e.g. using advanced training methods such as forced repetitions (Drinkwater, et al. 2007), pre-exhaustion (Fisher et al. 2014), or breakdown sets (Fisher et al. 2015)), or with differing muscle actions (Fisher et al. 2016b). Indeed, evidence has been presented that it might not be necessary to reach MF in order to optimise strength adaptations (Davies, et al. 2016). However, it is likely there exists a threshold for intensity effort, below which maximal recruitment of motor units and corresponding muscle fibres is not achieved (Fisher, et al. 2016). Our data, and this body of literature, suggests that recruiting all available motor units and muscle fibres through training to momentary failure, as per the size principle (Denny-brown and Pennybacker 1938; Carpinelli, 2008), is likely a primary driving force towards strength adaptations, and ensures that any threshold has been surpassed. Research has suggested that, for the same submaximal force demands, there is greater motor unit recruitment during muscle actions performed with increasing actual or intended velocities (Desmedt and Godaux 1977; Harwood and Rice 2012). This appears to be a result of reduced motor unit recruitment thresholds (Harwood and Rice 2012) and the fewer cross-bridges formed during faster muscle actions (Wilkie 1950). 
Repetition Duration in trained males and females

Indeed, previous publications have reported equivocal adaptations when considering repetition duration, however, these might have been compounded by parity in the number of muscle actions/repetitions performed and a disparity in training load and TUL (Keeler et al. 2001; Rana et al., 2008; Kim et al. 2011). However, the present study suggests that where load and effort are matched, the number of muscle actions/repetitions performed appears to be unimportant. It would seem fair to hypothesise that when muscle actions are performed to momentary failure there is likely very similar motor unit recruitment irrespective of the conditions under which they are performed (Potvin and Fuglevand 2017). However, since we did not measure motor unit recruitment and it has only been assumed to be maximal based on training to MF (Carpinelli, 2008), we should be cautious not to provide conclusions unsupported by our research design. Future research might consider the motor unit recruitment at equivalent loads using different repetition durations.

Body composition changes in participants from all groups were minimal, and likely within the margin of error for the measurement used (Collins et al. 2004; Dempster and Aitkins 1995). Previous research has reported large increases in regional CSA even without changes to body mass or body composition (e.g. Ivey et al. 2000). We also considered changes to arm and thigh muscle area though in these outcomes there were also no significant changes that exceeded measurement error. Previous authors have discussed that a plateau in muscle size adaptations is common in resistance trained individuals, and it is logical that a muscle does not continue to grow in size in accordance with continuation of a resistance training programme (Counts et al. 2017). In this sense, it is unsurprising that in our trained participants there was minimal change in muscle size. It is however plausible that morphological adaptations occurred at an architectural level within the muscle which were not detectable by our methods. For example, changes in muscle density might have occurred as a result of: i). changes in pennation angle of muscle fibres allowing an increased number of myofibrils, and/or, ii). an increase in muscle fibre size, neither of which necessarily correspond to changes in whole muscle CSA. However, this is purely speculative based on previous research where trained participants performed resistance exercise to MF (Morton, et al. 2016), and 
where positive strength changes occur in an absence of change to whole muscle CSA. It is also reasonable to consider that a lack of significant change to muscle size across each of the groups herein might be a result of insufficient training stimulus. Whilst the use of a low-volume (single set) protocol represents considerable time efficiency for strength increases, it might not be sufficient to produce meaningful increases in muscle mass in trained participants. Finally, we should also acknowledge that no effort was made to control or monitor dietary intake. Indeed, protein consumption might not have been sufficient to ensure muscle protein synthesis exceeded muscle protein breakdown, and thus; sustain continued growth in trained participants (Tipton and Wolfe, 2001). Whilst the lack of nutritional assessment is a potential limitation of the present study, adds ecological validity and is thus representative of the population group considered.

Finally, to our knowledge the present study is the first to consider fasted blood-glucose levels in trained participants. Previous research has reported that higher fasted blood glucose levels (e.g. $>85 \mathrm{mg} / \mathrm{dl}$ ) can be a risk factor for cardiovascular death (Bjørnholt et al. 1999). The present sample of 62 participants showed a pre-intervention mean fasted blood glucose level of $79.7 \pm 14.9$ $\mathrm{mg} / \mathrm{dl}$, within a normal range and not at elevated risk of cardiovascular death (Bjørnholt et al. 1999). These values are as expected from a group of resistance trained participants. However, fasted bloodglucose values showed a reduction to $75.7 \pm 16.1 \mathrm{mg} / \mathrm{dl}$ post-intervention. Whilst not statistically significant ( $p=0.082$ for post hoc pre-post paired samples $t$-test for combined sample) this might be clinically meaningful as it represents a shift from the top, to the bottom of the $2^{\text {nd }}$ quartile based on data from a large cohort (Bjørnholt et al. 1999). In this sense, whilst resistance training appears to help aid in blood glucose control and has the greatest impact in inactive/untrained, or prediabetic/diabetic populations (Mann et al. 2014), there might be meaningful improvement in the reduction of fasted blood glucose by the continuation of a resistance training programme in those already participating. 
Repetition Duration in trained males and females

The present study adds to the body of literature considering previously trained participants testing strength changes across multiple upper- and lower-body exercises, however, we should consider that this research is not without its limitations. Firstly, we did not include a non-training control group in the present study. This was justified in the study design due to the use of trained participants; it was deemed that a control condition was to continue with their traditional training programme rather than cease training completely. Future research might consider the impacts of trained participants ceasing resistance exercise for prolonged periods (e.g. 10 weeks). In addition each group performed the same training protocol every $4^{\text {th }}$ session meaning that 5 of 20 workouts were identical. This was deemed appropriate to maintain parity for load and to avoid any bias in practicing the repetition duration used for testing, however, it could be argued that this parity also meant the training protocols were not sufficiently different to produce differing adaptations. Secondly, our maximal strength data is based on predictive equations, which whilst showing a very high correlation to actual 1RM ( $r=0.99$; Nascimento et al. 2007) does not have the same validity as maximal testing performed using isometric or isokinetic dynamometry. Thirdly, as discussed, we did not monitor nutritional changes, or provide nutritional guidance, that might have impacted upon body composition. Recent research has advocated $1.6 \mathrm{~g} / \mathrm{kg}$ of protein to attain increases in muscle hypertrophy and participants might not have met these recommendations (Morton et al. 2017) potentially explaining the lack of changes in lean mass or muscle size. However, we elected not to encourage dietary changes to the participants of the present study since this might have also impacted blood-glucose levels. Finally, the present resistance training protocol included a lowvolume approach of a single-set per exercise. Many might argue that an absence of positive adaptations to muscle size might be a product of insufficient resistance training volume (e.g. Schoenfeld et al. 2017). However, without a group performing a larger volume in the present study we cannot be certain of potential adaptations. The present article supports that a low-volume approach is a time-efficient protocol that promotes high adherence levels, and supports significant strength increases within trained participants. Future research, might consider higher volumes 
Repetition Duration in trained males and females

and/or greater training frequencies, as well as considering the perceived effort and muscular discomfort associated with differing repetition durations.

Practical applications

Results from the present study suggest that considerable increases in muscular strength can be attained by the use of brief, infrequent, and uncomplicated RT, specifically in persons with previous RT experience. Furthermore, that repetition duration and the number of muscle actions performed does not impact strength adaptations. From a practical perspective the present study suggests that trainers and trainees can self-select repetition duration based on other variables such as range of motion of the exercise performed, orthopaedic conditions, or personal preference. It is perhaps noteworthy that the LST groups in the Tanimoto and Ishii (2006), and Tanimoto et al. (2008) studies showed lower peak diastolic blood pressure for the longer- compared to short- repetition duration training groups. As such, there might be specific reasons why performing a longer repetition duration might be more appropriate (e.g. hypertensive participants). The present study supports that a low-volume, time efficient approach to RT can continue to promote strength increases even in resistance trained persons.

\section{Acknowledgements}

The authors would like to acknowledge the research assistants involved in training participants. The authors declare that there are no conflicts of interest. 
Repetition Duration in trained males and females

\section{References}

Baker, J.S., Davies, B., Cooper, S.M., Wong, D.P., Buchan, D.S., and Kilgore, L. 2013. Strength and Body Composition Changes in Recreationally Strength-Trained Individuals: Comparison of One versus Three Sets Resistance-Training Programmes. BioMed. Res. Int. Article ID 615901. PMID: 24083231

Barbalho, M., Gentil, P., Izquierdo, M., Steele, J., Fisher, J., and de Azevedo Raiol, R. 2017. There are no non-responders to low or high resistance training volumes among older women. Exp. Gerontol. 99: 18-26. PMID: 28918362

Bjørnholt, J.V., Erikssen, G., Aaser, E., Sandvik, L., Nitter-Hauge, S., Jervell, J., et al. 1999. Fasting blood glucose: an underestimated risk factor for cardiovascular death. Results from a 22-year followup of healthy nondiabetic men. Diabetes. Care. 22(1): 45-49. PMID: 10333902

Bonde-Peterson, F., Mork. A.L., and Nielson, E. 1975. Local muscle blood flow and sustained contractions of human arm and back muscles. Eur. J. Appl. Physiol. Occup. Physiol. 34:43-50. PMID: 1149731

Brzycki, M. 1993. Strength testing: predicting a one-rep max from repetitions to fatigue. J. Phys. Ed. Rec. Dance. 64: 88-90.

Burd, N.A., Andrews, R.J., West, D.W., Little, J.P., Cochran, A.J., Hector, A.J., et al. 2012. Muscle time under tension during resistance exercise stimulates differential muscle protein sub-fractional synthetic responses in men. J. Physiol. 15;590(2): 351-362. PMID: 22106173

Carpinelli, R.N. 2008. The size principle and a critical analysis of the unsubstantiated heavier-isbetter recommendation for resistance training. J. Exerc. Sci. Fit. 6(2): 67-86.

Cohen, J. 1992. A power primer. Psychol. Bull. 112: 155-159. PMID: 19565683

Collins, M.A., Millar-Stafford, M.L., Evans, E.M., Snow, T.K., Cureton, K.J., and Rosskopf, L.B. 2004. Effect of race and musculoskeletal development on the accuracy of air plethysmography. Med. Sci. Sports. Exerc. 36(6): 1070-1077. PMID: 15179179

Counts, B.R., Buckner, S.L., Mouser, J.G., Dankel, S.J., Jessee, M.B., Mattocks, K.T., et al. 2017. Muscle growth: To infinity and beyond? Muscle. Nerve. 56(6): 1022-1030. PMID: 28543604

Davies, T., Orr, R., Halaki, M., and Hackett, D. 2016. Effect of training leading to repetition failure on muscular strength: A systematic review and meta-analysis. Sports. Med. 46(4): 487-502. PMID: 26666744

Dempster, P., and Aitkens, S. 1995. A new air displacement method for the determination of human body composition. Med. Sci. Sports. Exerc. 27(12): 1692-1697. PMID: 8614327

Denny-Brown, D., and Pennybacker, J.B. 1938. Fibrillation and fasciculation in voluntary muscle. Brain. 61: 311-312.

Desmedt, J.E., and Godaux, E. 1977. Ballistic contractions in man: characteristic recruitment pattern of single motor units of the tibialis anterior muscle. J. Physiol. 264(3): 673-693. PMID: 845820

Drinkwater, E.J., Lawton, T.W., McKenna, M.J., Lindsell, R.P., Hunt, P.H., and Pyne, D.B. 2007. Increased number of forced repetitions does not enhance strength development with resistance training. J. Strength. Cond. Res. 21(3): 841-847. PMID: 17685709 
Fisher, J., Blossom, D., and Steele, J. 2016. A comparison of volume equated knee extensions to failure or not to failure upon rating of perceived exertion and strength adaptations. Applied. Physiol. Nutr. Metab. 41(2): 168-174. PMID: 26789094

Fisher, J.P., Carlson, L., and Steele, J. 2015. The effects of breakdown set resistance training on strength and body composition in young males and females. J. Strength. Cond. Res. 30(5): 14251432. PMID: 26439789

Fisher, J.P., Carlson, L., Steele J., and Smith, D. 2014. The effects of pre-exhaustion, exercise order, and rest intervals in a full-body resistance training intervention. Appl. Phys. Nutr. Metab. 39(11); 1265-1270. PMID: 25092528

Fisher, J., Steele, J., McKinnon, P., and McKinnon, S. 2014b. Strength gains as a result of brief, infrequent resistance exercise in older adults. J. Sports. Med. Article ID 731890.

Fisher, J., Steele, J., and Smith, D. 2017. High- and Low-Load Resistance Training: Interpretation and Practical Application of Current Research Findings. Sport. Med. 47(3): 393-400. PMID: 27480764

Fisher, J.P., Carlson, L., and Steele, J. 2016b. The effects of muscle action, repetition duration, and loading strategies of a whole-body, progressive resistance training programme on muscular performance and body composition in trained males and females. Appl. Phys. Nutr. Metab. 41(10):1064-1070. PMID: 27690566

Fisher, J.P., Ironside, M., and Steele, J. 2017b. Heavier and lighter load resistance training to momentary failure produce similar increases in strength with differing degrees of discomfort. Muscle. Nerve. 56:797-803. PMID: 28006852

Goto, K., Takahashi, K., Yamamoto, M., and Takamatsu, K. 2008. Hormone and recovery responses to resistance exercise with slow movement. J. Physiol. Sci. 58(1):7-14. PMID: 18186955

Harwood, B., and Rice, C.L. 2012. Changes in motor unit recruitment thresholds of the human anconeus muscle during torque development preceding shortening elbow extensions. J.

Neurophysiol. 107(10): 2876-2884. PMID: 22378176

Heymsfield, S.B., McManus, C., Smith, J., Stevens, V., and Nixon, D.W. 1982. Anthropometric measurement of muscle mass: revised equations for calculating bone-free arm muscle mass. Am. J. Clin. Nutr. 36: 680-690. PMID: 7124671

Hopkins, W.G. 2015. Spreadsheets for Analysis of Validity and Reliability. Sportscience. 19:36-42.

Housh, D.J., Housh, T.J., Weir, J.P., Weir, L.L., Johnson, G.O., and Stout, J.R. 1995. Anthropometric estimation of thigh muscle cross-sectional area. Med. Sci. Sports. Exerc. 27(5): 784-791. PMID: 7674885

Ivey, F.M., Roth, S.M., Ferrell, R.E., Tracy, B.L., Lemmer, J.T., Hurlbut, D.E., et al. 2000. Effects of age, gender, and myostatin genotype on the hypertrophic response to heavy resistance strength training. J. Gerontol. Med. Sci. 55(11): M641-748. PMID: 11078093

Keeler, L.K., Finkelstein, L.H., Miller, W., and Fernhall, B. 2001. Early-phase adaptations of traditionalspeed vs. superslow resistance training on strength and aerobic capacity in sedentary individuals. J. Strength. Cond. Res. 15(3): 309-314. PMID: 11710656

Kim, E., Dear, A., Ferguson, S.L., Seo, D., and Bemben, M.G. 2011. Effects of 4 weeks of traditional resistance training vs. superslow strength training on early phase adaptations in strength, flexibility, 
Repetition Duration in trained males and females

and aerobic capacity in college-aged women. J. Strength. Cond. Res. 25(11):3006-3013. PMID: 21993022

Loenneke, J.P., Wilson, G.L., and Wilson, J.M. 2010. A mechanistic approach to blood flow occlusion. Int. J. Sports. Med. 31(1): 1-4. PMID: 19885776

Mann, S., Beedie, C., Balducci, S., Zanuso, S., Allgrove, J., Bertiato, F., et al. 2014 Changes in insulin sensitivity in response to different modalities of exercise: a review of the evidence. Diabetes. Metab. Rex. Rev. 30(4): 257-268. PMID: 24130081

Mattocks, K.T., Buckner, S.L., Jessee, M.B., Dankel, S.J., Mouser, J.G., and Loenneke, J.P. 2017 Practicing the test produces strength equivalent to higher volume training. Med. Sci. Sports. Exerc. 49(9): 1945-1954. PMID: 28463902

Maughan, R., Leiper, J., and Greaves, M. 2011. Haemotology. In: Kinanthropometry and Exercise Physiology Laboratory Manual: Tests, Procedures and Data. 2nd ed. Eston R, Reilly R. Routledge, Abingdon, Oxon.

Morton, R.W., Murphy, K.T., McKEllar, S.R., Schoenfeld, B.J., Henselmans, M., Helms, E., et al. 2017. A systematic review, meta-analysis and meta-regression of the effect of protein supplementation on resistance training-induced gains in muscle mass and strength in healthy adults. Br. J. Sports. Med. 52(6): 376-384. PMID: 28698222

Morton, R.W., Oikawa, S.Y., Wavell, C.G., Mazara, N., McGlory, C., Quadrilatero, J., et al. 2016. Neither load nor systemic hormones determine resistance training-mediated hypertrophy or strength gains in resistance-trained young men. J. Appl. Physiol. 121(1): 129-138. PMID: 27174923

Nascimento, M.A. do, Cyrino E.S., Nakamura F.Y., Romanzini, M., Pianca H.J.C., and Queiróga, M.R. 2007. Validation of the Brzycki equation for the estimation of 1-RM in the bench press. Rev. Bras. Med. Esporte. 13(1): 40e-42e.

Potvin, J.R., and Fuglevand, A.J. 2017. A motor unit-based model of muscle fatigue. PLoS. Comput. Biol. 13(6): e1005581. PMID: 28574981

Rana, S.R., Chleboun, G.S., Gilders, R.M., Hagerman, F.C., Herman, J.R., Hikida, R.S., et al. 2008. Comparison of early phase adaptations for traditional strength and endurance, and low velocity resistance training programs in college-aged women. J. Strength. Cond. Res. 22: 119-127. PMID: 18296964

Schoenfeld, B.J., Ogborn, D., and Krieger, J.W. 2017. Dose-response relationship between weekly resistance training volume and increases in muscle mass: A systematic review and meta-analysis. J. Sports. Sci. 35(11): 1073-1082. PMID: 27433992

Schoenfeld, B.J., Ratamess, N.A., Peterson, M.D., Contreras, B., and Tiryaki-Sonmez, G. 2015. Influence of Resistance Training Frequency on Muscular Adaptations in Well-Trained Men. J. Strength. Cond. Res. 29(7): 1821-1829. PMID: 25932981

Schoenfeld, B.J. 2010. The mechanisms of muscle hypertrophy and their application to resistance training. J. Strength. Cond. Res. 24(10): 2857-2872. PMID: 20847704

Schuenke, M.D., Herman, J.R., Gliders, R.M., Hagerman, F.C., Hikida, R.S., Rana, S.R., et al. 2012. Early-phase muscular adaptations in response to slow-speed versus traditional resistance-training regimens. Eur. J. Appl. Physiol. 112: 3585-3595. PMID: 22328004 
Silliman, K., Rodas-Fortier, K., and Neyman, M. 2004. A survey of dietary and exercise habits and perceived barriers to following a healthy lifestyle in a college population. Cal. J. Health. Prom. 18: 281

Steele. J., Fisher, J., Giessing, J., and Gentil, P. 2017. Clarity in Reporting Terminology and Definitions of Set End Points in Resistance Training. Muscle. Nerve. 56(3): 368-374. PMID: 28044366

Steele, J., Fisher, J., Skivington, M., Dunn, C., Arnold, J., Tew, G., et al. 2017b. A higher effort-based paradigm in physical activity and exercise for public health: making the case for a greater emphasis on resistance training. BMC. Public. Health. 17: 300. PMID: 28381272

Tanimoto, M., and Ishii, N. 2006. Effects of low-intensity resistance exercise with slow movement and tonic force generation on muscular function in young men. J. Appl. Physiol. 100: 1150-1157. PMID: 16339347

Tanimoto, M., Sanada, K., Yamamoto, K., Kawano, H., Gando, Y., Tabata, I., et al. 2008. Effects of whole-body low-intensity resistance training with slow movement and tonic force generation on muscular size and strength in young men. J. Strength. Cond. Res. 22(6): 1926-1938. PMID: 18978616

Tipton, K.D., and Wolfe, R.R. 2001. Exercise, protein metabolism, and muscle growth. Sport. Nutr. Exerc. Metab. 11(1): 109-132. PMID: 11255140

Trost, S.G., Owen, N., Bauman, A.E., Sallis, J.F., and Brown, W. 2002. Correlates of adults' participation in physical activity: Review and update. Med. Sci. Sports. Exerc. 34(12): 1996-2001. PMID: 12471307

Watanabe, Y., Tanimoto, M., Ohgana, A., Sanada, K., Miyachi, M., and Ishii, N. 2013. Increased muscle size and strength from slow-movement, low-intensity resistance exercise and tonic force generation. J. Aging. Phys. Act. 21: 71-84. PMID: 22832536

West, D.W., and Phillips, S.M. 2012. Associations of exercise-induced hormone profiles and gains in strength and hypertrophy in a large cohort after weight training. Eur. J. Appl. Physiol. 112(7): 26932702. PMID: 22105707

Westcott, W.L., Winett, R.A., Anderson, E.S., Wojcik, J.R., Loud, R.L.R., Cleggett, E., et al. 2001. Effects of regular and slow speed resistance training in muscle strength. J. Sports. Med. Phys. Fit. 41: 154-158. PMID: 11447355

Whitley, E., and Ball, J. 2002. Statistics review 4: sample size calculations. Crit. Care. 6: 335-341. PMID: 12225610

Wilkie, D.R. 1950. The relation between force and velocity in human muscle. J. Physiol. 110:249-280. PMID: 15406429

Winett, R.A., Williams, D.M., and Davy, B.M. 2009. Initiating and maintaining resistance training in older adults: A social cognitive theory-based approach. Br. J. Sports. Med. 43(2): 114-119. PMID: 18628361 
Repetition Duration in trained males and females

\section{Figure Legends}

Figure 1: Mean ( $\pm 95 \% \mathrm{Cls})$ change in predicted $1 \mathrm{RM}$ for chest press, leg press, and pull down exercises as well as combined total for each group. 
Table 1. Weekly resistance training programme, workout A and B were both performed once each week

\begin{tabular}{cc} 
Workout A & Workout B \\
\hline Chest press & Chest press \\
Leg Press & Leg Press \\
Pull-Down & Pull-Down \\
Pullover & Pec-fly \\
Hip adduction & Elbow flexion \\
Hip abduction & Knee flexion \\
Heel raise & Knee extension \\
Abdominal flexion & Ankle dorsi-flexion \\
Lumbar extension & Torso rotation
\end{tabular}


Table 2. Participant demographics and pre- and post-intervention predicted $1 \mathrm{RM}(\mathrm{kg})$. All data is given as mean ( $\pm S \mathrm{SD})$.

\begin{tabular}{|c|c|c|c|}
\hline & $2 s: 4 s$ & 10s:10s & $30 \mathrm{~s}: 30 \mathrm{~s}: 30 \mathrm{~s}$ \\
\hline Age (years) & $39 \pm 14$ & $39 \pm 10$ & $42 \pm 14$ \\
\hline Stature $(\mathrm{cm})$ & $171.3 \pm 8.1$ & $172.4 \pm 9.3$ & $168.9 \pm 10.0$ \\
\hline Body mass (kg) & $75.4 \pm 14.2$ & $79.2 \pm 14.4$ & $71.6 \pm 13.1$ \\
\hline \multicolumn{4}{|l|}{ Pre intervention $1 \mathrm{RM}(\mathrm{kg})$} \\
\hline Chest press & $57.1 \pm 25.0$ & $62.9 \pm 27.2$ & $64.1 \pm 22.8$ \\
\hline Leg press & $126.9 \pm 58.5$ & $135.5 \pm 58.0$ & $139.2 \pm 44.5$ \\
\hline Pulldown & $183.4 \pm 62.9$ & $202.1 \pm 63.3$ & $205.5 \pm 55.9$ \\
\hline \multicolumn{4}{|l|}{ Post-Intervention 1RM (kg) } \\
\hline Chest press & $61.5 \pm 24.5^{*}$ & $65.8 \pm 27.5^{*}$ & $69.5 \pm 22.5^{*}$ \\
\hline Leg press & $143.3 \pm 58.6^{*}$ & $145.2 \pm 58.9^{*}$ & $153.3 \pm 45.7^{*}$ \\
\hline Pulldown & $195.9 \pm 62.9^{*}$ & $213.1 \pm 64.9^{*}$ & $220.6 \pm 57.1^{*}$ \\
\hline
\end{tabular}

*Indicates all groups resulted in significant pre- to post intervention change for each exercise as determined by $95 \%$ Cls for estimated marginal means (see Figure 1); Note: There were no significant differences between groups for change in muscular performance for any exercise. 
Table 3. Estimated marginal means $(95 \% \mathrm{Cls})$ showing no significant changes, or between group differences, in anthropometric outcomes.

\begin{tabular}{lccc}
\hline & \multicolumn{1}{c}{$10 \mathrm{~s}: 10 \mathrm{~s}$} & $30 \mathrm{~s}: 30 \mathrm{~s}: 30 \mathrm{~s}$ & $2 \mathrm{~s}: 4 \mathrm{~s}$ \\
\hline Body fat $(\%)$ & $0.08(-0.64$ to 0.80$)$ & $0.16(-0.58$ to 0.90$)$ & $-0.29(-1.09$ to 0.50$)$ \\
Lean mass $(\mathrm{kg})$ & $0.09(-1.08$ to 1.25$)$ & $-0.55(-1.75$ to 0.65$)$ & $1.07(-0.19$ to 2.34$)$ \\
Arm MA $\left(\mathrm{cm}^{2}\right)$ & $-1.18(-3.65$ to 1.29$)$ & $1.63(-1.04$ to 4.31$)$ & $0.28(-2.41$ to 2.97$)$ \\
& $-0.69(-4.72$ to 3.34$)$ & $3.16(-1.09$ to 7.41$)$ & $3.48(-0.90$ to 7.76$)$ \\
\hline
\end{tabular}




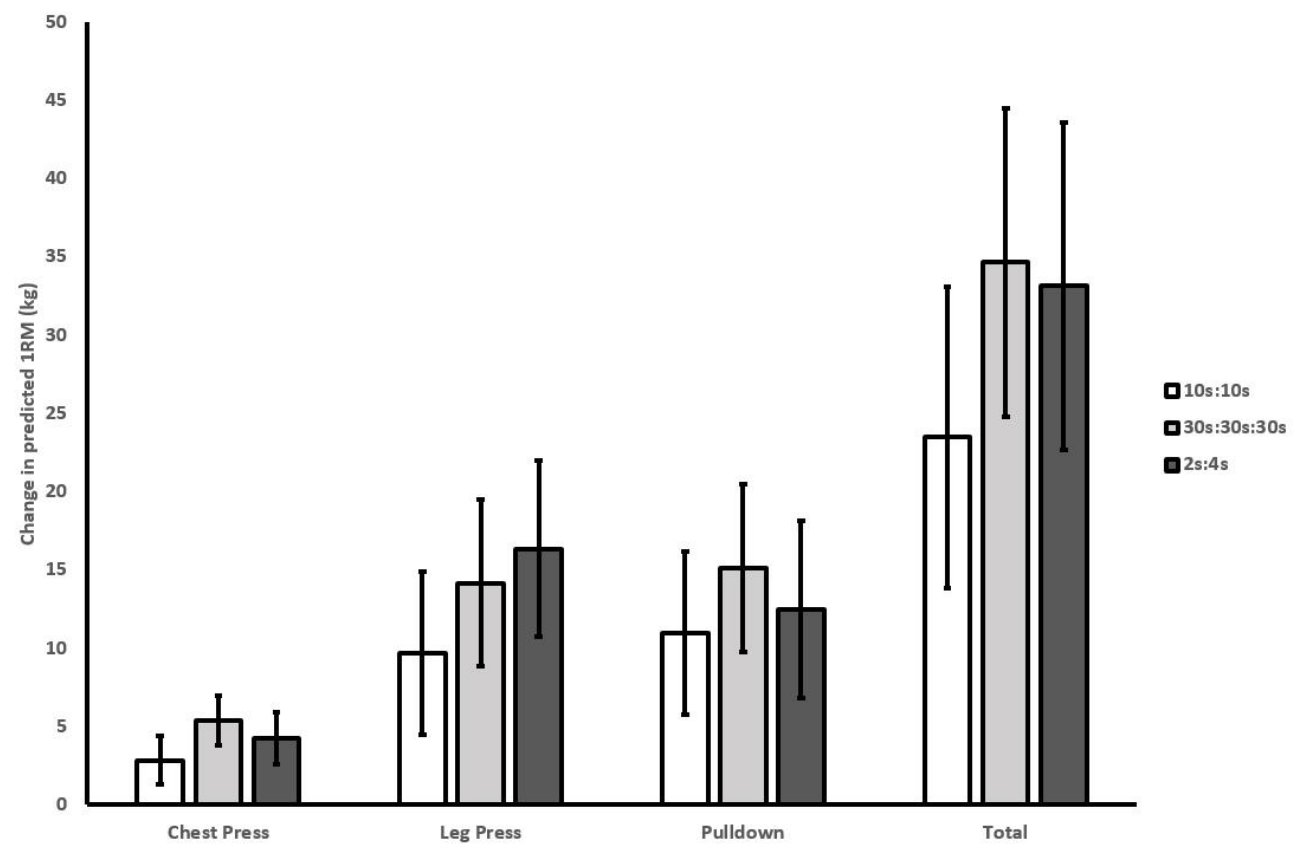

Figure 1: Mean ( $\pm 95 \%$ CIs) change in predicted 1RM for chest press, leg press, and pull down exercises as well as combined total for each group.

$93 \times 61 \mathrm{~mm}(300 \times 300 \mathrm{DPI})$ 